\title{
Dense Interest Points
}

\author{
Tinne Tuytelaars \\ K.U.Leuven, ESAT - PSI \\ Tinne. Tuytelaarsdesat.kuleuven.be
}

\begin{abstract}
Local features or image patches have become a standard tool in computer vision, with numerous application domains. Roughly speaking, two different types of patchbased image representations can be distinguished: interest points, such as corners or blobs, whose position, scale and shape are computed by a feature detector algorithm, and dense sampling, where patches of fixed size and shape are placed on a regular grid (possibly repeated over multiple scales). Interest points focus on 'interesting' locations in the image and include various degrees of viewpoint and illumination invariance, resulting in better repeatability scores. Dense sampling, on the other hand, gives a better coverage of the image, a constant amount of features per image area, and simple spatial relations between features. In this paper, we propose a hybrid scheme, which we call dense interest points, where we start from densely sampled patches yet optimize their position and scale parameters locally. We investigate whether doing so it is possible to get the best of both worlds.
\end{abstract}

\section{Introduction}

Recent progress in the field of object and scene recognition has shown that good image representations are crucial when it comes to learning high level concepts from images. Even the best learning mechanisms cannot easily make up for bad features, also known as the 'garbage in, garbage out' principle. In this paper, we inspect two of the most commonly used low-level image representations: interest points and dense sampling on a regular grid. We look at their respective strengths and weaknesses, and propose a hybrid scheme, dense interest points, combining the best of both worlds.

When the goal is to recognize specific objects or to find correspondences between two or more wide baseline views of the same scene, viewpoint invariant interest points, such as corners or blobs are to be preferred (see [22] for a recent survey of these methods). This is also the application domain for which interest point detectors were originally developed. Later, the same interest point detectors have also been applied successfully in the context of categorylevel object recognition, scene classification and image understanding (e.g. [7, 9, 19]). However, more and more researchers have switched to a much simpler scheme recently, which turns out to work just as well in this new context, namely dense sampling of the image with a regular grid, possibly over a range of scales (e.g. [6, 20,23]). Nowak et al. [4] have shown that in the context of category-level object recognition the density of extracted features is the dominant factor in determining the recognition performance, while the repeatability of the individual features (in their study interest points versus random patches) plays only a secondary role. In the recent Pascal Visual Object Classes Challenges [5], it has been shown that the best performance is obtained by using a combination of both interest points and densely sampled image patches.

Interest points yield a high repeatability, i.e. they can be extracted reliably and are often found again at similar locations in other images of the same object or scene. Also, with interest points the user can choose the appropriate level of viewpoint and illumination invariance, depending on the application and expected variability. As the name suggests, interest point detectors focus on 'interesting' regions, which are typically regions with high information content, that can be localized precisely. These are in some sense optimal when the goal is to find correspondences between two views of the same object or scene. However, when the goal is image interpretation, it is unclear whether the heuristics on which these feature extraction methods are based actually perform a good feature selection task or not.

On the downside, the number of interest points extracted from an image varies a lot based on the image content - often from a few hundred to several thousands without changing the parameters. Sometimes, when the contrast in an image is low, not a single interest point is found, making the image representation useless.

Dense sampling on a regular grid, on the other hand, results in a good coverage of the entire object or scene and a constant amount of features per image area. Regions with less contrast contribute equally to the overall image rep- 


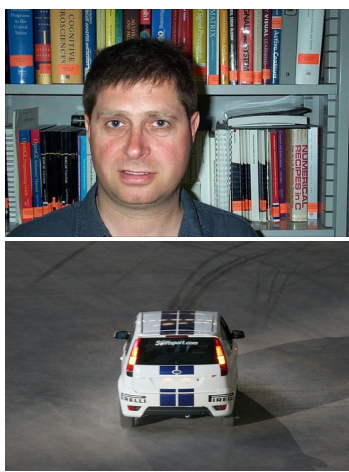

original image

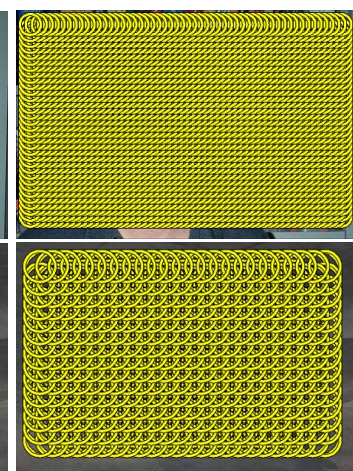

dense sampling

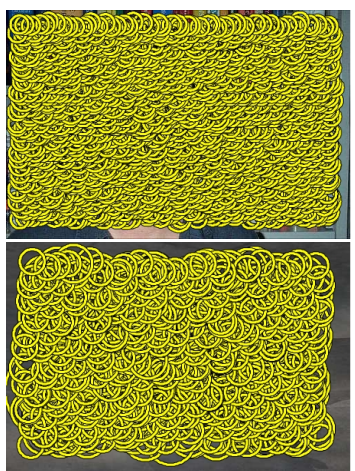

dense interest points

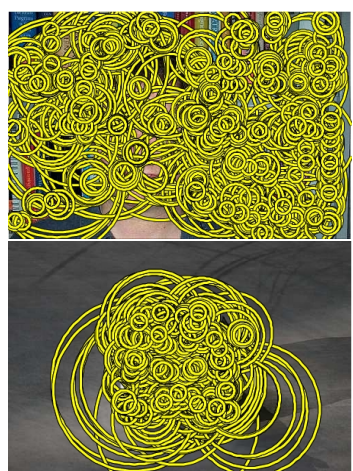

interest points

Figure 1. Dense interest points, as proposed in this paper, (middle) form a hybrid scheme in between dense sampling on a regular grid (left) and interest point detection (right), combining (to some extent) the good coverage and (semi)regularity of dense sampling with the repeatability of interest points.

resentation. This is based on the idea that, even if such patches cannot be matched accurately, they do contain valuable information regarding the image content, that may help to interpret the scene. Also, spatial relations between features follow a regular pattern that is easily represented in a simple model. This is important when using Markov or conditional random fields or when modelling the spatial configuration of features. This does not hold for interest points, where spatial relations are more arbitrary and, as a consequence, incorporating information on the spatial configuration is more complicated. For instance, consider the definition of the concept of 'neighbours'. Sivic et al. have proposed taking the $N$ nearest neighbours [18], but these are in some cases quite far away. Alternatively, one can use all interest points within a certain radius around the given interest point, but then the number of neighbours can vary greatly.

On the downside, dense sampling cannot reach the same level of repeatability as obtained with interest points, unless sampling is performed extremely densely - but then the number of features quickly grows unacceptably large. In practice, researchers often use an overlap between patches of $50 \%$ or less. This is clearly not enough to guarantee similar descriptors in case of structured scenes, especially when combined with SIFT-like descriptors [12], which further divide the region in smaller subpatches (in spite of SIFTs robustness to small misalignments).

Dense Interest Points, i.e. the hybrid scheme proposed in this paper, is our attempt to combine the advantages of both schemes. We start from densely sampled image patches and then apply for each feature a local optimization of the position and scale within a bounded search area. This way, we get dense coverage of the entire scene and clearly defined spatial relations, as in the case of dense sampling, yet with improved repeatability, as in the case of interest points (see also figure 1).
Related Work To the best of our knowledge, no hybrid feature extraction schemes combining ideas from interest point detection with ideas from dense sampling have been proposed to date in the context of object recognition or image understanding. When searching for stereo correspondences, however, it is common practice to divide the image in a small number of subareas and adapt the threshold of the interest point detector so as to ensure that a similar number of features is found in each subarea (e.g. [16]). This guarantees that the features are well distributed over the entire image, which results in a more accurate camera calibration. The dense interest points proposed here can be considered as an extreme case of this idea, where the subareas have been made so small they each contain one and only one feature, and with the extension that also the scale dimension is taken into account. It is only in this extreme case that the benefits of preserving the (semi-)regular grid structure appear.

Similarly, in their image stitching work, Brown et al. [2] introduce the concept of adaptive non-maximal suppression. Instead of selecting features based on a threshold on the corner strength, they increase the radius of the nonmaximal suppression, avoiding nearby detections and hence resulting in a better distribution of the features over the image. Still, large portions of the image may not have a single feature, and again there is no (semi-)regularity in the feature distribution.

Tola et al. have proposed a scheme for efficiently computing dense descriptors [20]. However, they do not change the detection scheme, sticking to the traditional sampling on a regular grid. So their work can be seen as complementary to ours.

Finally, apart from regular grids, some authors have proposed to use random sampling (e.g. [13, 4]), but this does not seem to have any advantage over regular grids, and equally ignores the actual image content during the feature 
extraction process.

The rest of the paper is organized as follows. First, we describe in more detail how we extract dense interest points and discuss their main properties (section 2). Next, we experimentally validate the usefulness of such features, both with respect to repeatability as well as in the context of a classification and matching application (section 3). Section 4 concludes the paper.

\section{Dense Interest Points}

The starting point for our dense interest points consists of a set of patches densely sampled on a regular grid over multiple scales. For larger scales, a coarser grid is used, such that the overlap ratio between neigbouring patches remains (approximately) fixed. This can also be thought of as applying the same grid to multiple downscaled versions of the original image in a scalespace pyramid.

Next, each of the patches so obtained is further refined, both with respect to its location as with respect to its scale. To this end, any measure of 'interestingness' proposed in the context of interest point detection can be used, such as the Harris cornerness measure, the determinant of the Hessian, the Laplacian, or saliency based on entropy. The refinement consists of searching for a local maximum within a bounded search area. This search area reaches up to the boundaries of the search areas of the neighbouring patches, both spatially as well as over scales. This gives us the maximal freedom to adapt to the image content while still keeping the topological structure of the original regular grid intact.

In our implementation, we have chosen the Laplacian as optimization criterion. When the goal is to find stable features, the Laplacian is often criticized because it finds too many features on edges, which are not well localized. However, in an image understanding context, the goal is to find representative features rather than stable ones. If within a bounded search area an edge is the most prominent feature, we want the refinement process to center the patch onto this edge, even if this results in an unstable localization. Hence the Laplacian seems to be a good choice.

Of course, it cannot be guaranteed that a true local maximum is found within such a limited search area, e.g. if the function to be maximized is monotonously increasing. In such cases, a point on the boundary of the search area is selected. One may argue that this is suboptimal, since it depends on the discretization imposed by the underlying regular grid. However, it is not any worse than selecting the center point, as is done when sampling patches on a regular grid without refinement. If the function to be maximized is constant over the entire search area, the refinement process selects the center point (or it is determined randomly, based on the noise in the signal).

\subsection{Implementation details}

In our implementation, we deviated slightly from the traditional scale space pyramid. Within each octave, we keep the size of the images constant and vary the sigma of the Laplacian-of-Gaussian instead. This results in rectangular search areas and hence a slightly simpler implementation. However, note that this is just an implementation choice and not inherent to the use of dense interest points. Figure 2 sketches the core of the algorithm and its relation to the standard Laplacian-of-Gaussians (LoG) scheme as well as to dense sampling on a regular grid. First, we build a LoG scale pyramid. Here, we typically use more pyramid levels per octave than usual (16 in our experiments). The standard LoG feature extraction process then checks for each pixel whether it is a local maximum in a $3 \times 3 \times 3$ neighbourhood (spatially and over scales). Only if a true local maximum is found, is the pixel retained as interest point (and further refined using parabolic interpolation). This process is repeated for each pixel, each time checking whether it is a local maximum in a small $(3 \times 3 \times 3)$ neigbourhood. Instead, we look for maxima in larger search areas, spanning multiple scales and extending beyond the direct neighbouring pixels. The size of these search areas is determined based on the underlying regular grid: each search area corresponds to the Voronoi cell around a vertex of the regular grid. For instance, when using a grid consisting of patches at the lowest scale of size $32 \times 32$ with $50 \%$ overlap between patches and 2 scales per octave, the distance between two neighbouring vertices is 16 pixels and the search area is 16 pixels $\times 16$ pixels $\times 8$ scale levels (assuming 16 scale levels per octave). However, in contrast to the standard LoG scheme, the maximum found within such a search area is always retained as dense interest point, even if it is not a local maximum. This may happen for maxima found on the boundary of the search region. Similarly, even if there are multiple local maxima within the search area, only one dense interest point per search area is retained. As a result, even though our search areas are much larger and nonoverlapping, we typically find a larger number of features. In fact, the number of features is fixed and corresponds to the number of vertices of the regular grid.

With more scale levels per octave, we can determine the scale of the dense interest points accurately, without having to rely on parabolic interpolation.

It is important to stress here that the proposed method is by no means limited to this framework of a Laplacian of Gaussians pyramid. As mentioned before, any interest point detection method that is based on finding a local maximum of a measure for interestingness can be extended to dense interest point detection. 

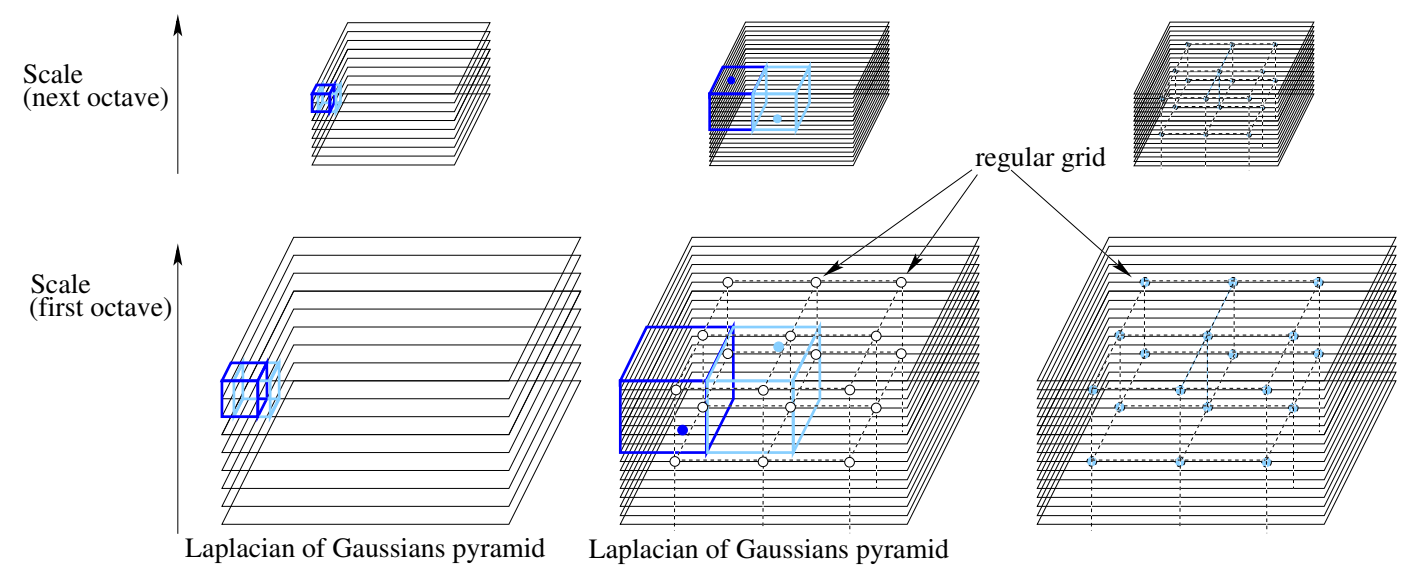

Figure 2. Illustration how dense interest points can be computed using a Laplacian-of-Gaussians scale pyramid. Local maxima are searched within larger neighbourhoods than the $3 \times 3 \times 3$ neighbourhoods used by standard interest point detection schemes. Moreover, we always keep exactly one feature per search neighbourhood. Instead of LoG, other interest measures can be used as well.
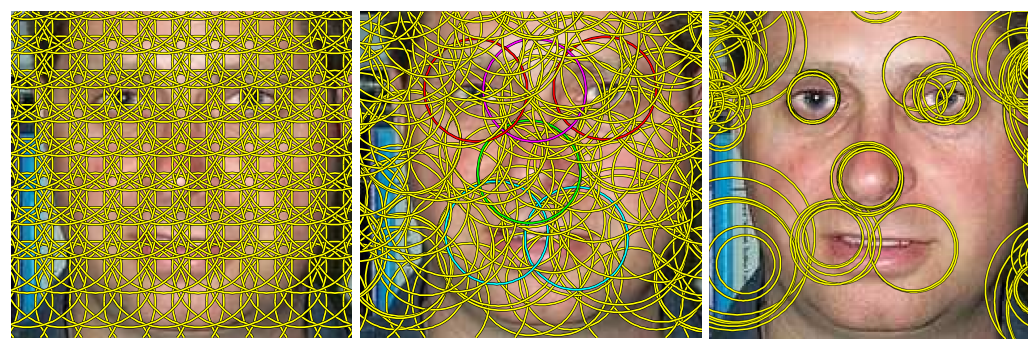

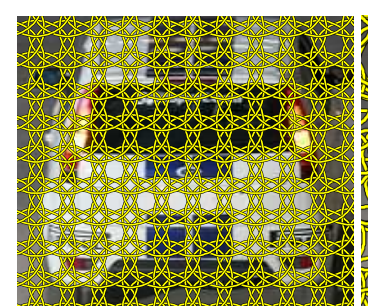

dense sampling

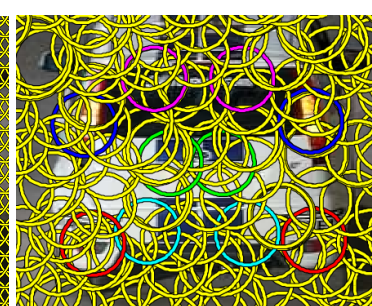

dense interest points

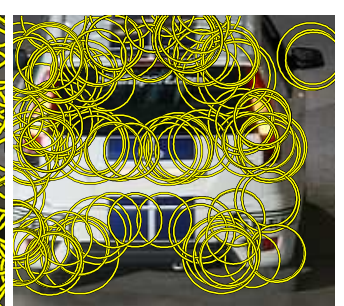

interest points

Figure 3. Unlike dense sampling on a regular grid (left) dense interest points (middle), like standard interest points (right), adapt to the underlying image structure, so they can center on important features in the image, like the eyes, nose tip, nose ridge and mouth corners of the face (top) or the lights or prints of the car (bottom) (highlighted using colour coding). Likewise, this adaptation ensures that similar patches are extracted on the two halves of these symmetric objects (zoomed in and only a single scale layer for clarity).

\subsection{Discussion}

Figure 3 shows some of the patches extracted both with and without this refinement step, i.e. using the newly proposed dense interest points and based on dense sampling on a regular grid, as well as the result of a standard interest point detector (Hessian Laplace [14]). As can be seen from the figure, the configuration of dense interest points is less regular than the result of dense sampling, yet they still densely cover the entire image in contrast to the Hessian
Laplace features, which for the first example focus more on the background than on the foreground object. Moreover, when there is a prominent feature in the image, such as the eyes, nose tip, nose ridge, or mouth corners for the face, or the lights or prints for the car, there is a good chance that one of the dense interest points locks onto it. Note that this also holds for the nose ridge, which was not found with the standard interest point detector, due to the lack of contrast. As a result, similar features are found for both halves of these symmetric objects (see also figure 4). From these ob- 


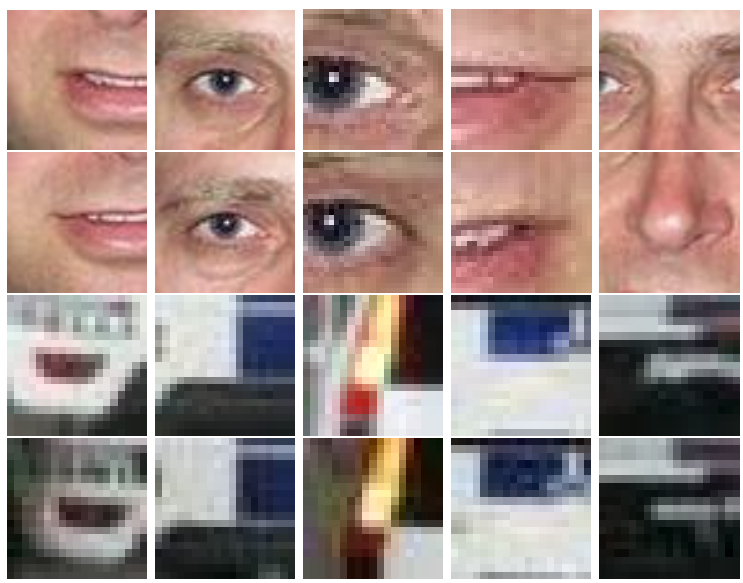

Figure 4. Some example patches extracted with dense interest points (with some of the second and fourth row patches mirrored, to highlight the similarity between patches extracted from symmetric locations).

servations, it can be expected that also for different views of the same object a higher repeatability is obtained.

Some statistics Closer inspection of the refinement procedure at work reveals that only for a very small fraction of the features a true local maximum over space and scale is found (on the order of one percent, depending on the image content and the density of the grid). For another two out of every three features, approximately, a local maximum over space is reached, but not over scale. For the remaining 30\%, the refinement procedure yields a feature on the boundary of the search area. However, this also depends on the relative scale of the sigma of the LoG relative to the size of the search neighbourhoods. We expect that with a smaller value for sigma, more true local maxima will be found.

Spatial structure Figure 5 shows some examples of the original regular grid (left) and the deformed version after refinement (middle), with vertices corresponding to the centers of features. The topological structure is preserved, even though the grid itself is deformed, adapting to the actual image content. Sometimes, two vertices end up very close to each other. This happens when a local maximum is located on or near a boundary between two search areas. Further note how the refinement procedure tries to align the grid vertices with interesting structures in the image, including not only blobs, but also edges, ridges, and symmetry axes. Even though we only show the grid for a single scale level, it is actually a 3D grid structure in a scalespace volume, also linking overlapping patches at different scale levels.

Since the topological structure of the original regular grid is kept intact, spatial relations such as 'left of', 'right of', 'above', 'below', '4-neighbours' or '8-neighbours' with respect to the grid are preserved and can be exploited e.g. when using a Markov or conditional random field. Also when modelling spatial configurations in general, such grid structure can be useful. For instance, one can define pairs of features that are separated by a fixed number of edge segments $-e . g$. in figure 5, the mouth corner and eye are above each other and four grid lengths apart from each other and together can form an interesting, more discriminative feature (e.g. in a freqent itemset mining context as in [21]).

So far, we looked at the grid as providing links or spatial relations between features, but one can also consider the 'patches' formed by the quadrangles of the grid. These are somewhat reminiscent of superpixels [17], albeit not based on color segmentation and preserving the regular structure we also find with normal pixels.

When using standard interest points, one can approximate these results by computing a Delaunay triangulation (see figure 5, right). However, the obtained triangles vary a lot in scale and are sometimes very elongated, linking far away points. There is also no regularity in the obtained grid structure in this case.

\section{Experimental Evaluation}

We validate the usefulness, strengths and weaknesses of the newly proposed image representation in three experimental setups. First, we evaluate the repeatability under scale, rotation and viewpoint variations. Next, we evaluate the new features in the context of a classification and matching task.

For all these experiments, we use the same set of parameters. We start from a regular grid with $50 \%$ overlap between neighbouring patches and two scales per octave, with the smallest scale corresponding to $32 \times 32$ patches and increasing scales over 4 octaves. For a typical Pascal VOC challenge image, this results in around 1000 features per image. The optimization criterion for the refinement step is the Laplacian. Features are described with SIFT [12], with one tiny modification: we only normalize the features if the original norm is larger than one, otherwise, we keep the feature descriptor without normalization. This is necessary to avoid blowing up noise for features extracted on homogeneous image areas.

\subsection{Repeatability}

First, we evaluate the repeatability of the newly proposed features. To this end, we use some of the standard benchmarking sequences as well as the evaluation protocol proposed and made available by [15]. We compare our dense interest points with patches extracted by dense sampling on the same regular grid, and with the Hessian-Laplace scaleinvariant interest point detector of [14]. The results are shown in figure 6 . Clearly, dense interest points do not reach 


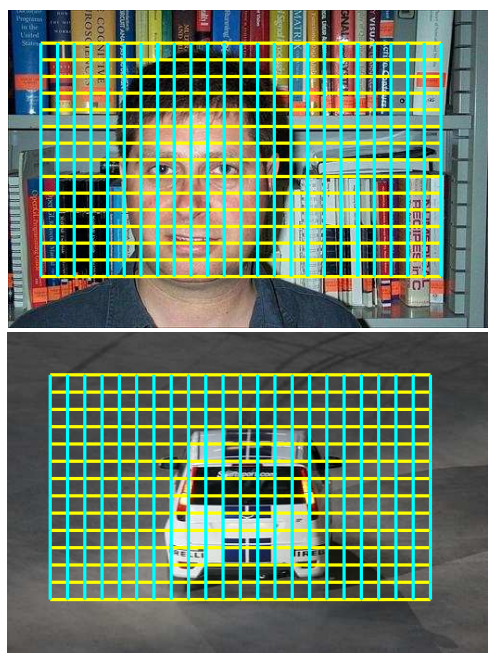

dense sampling

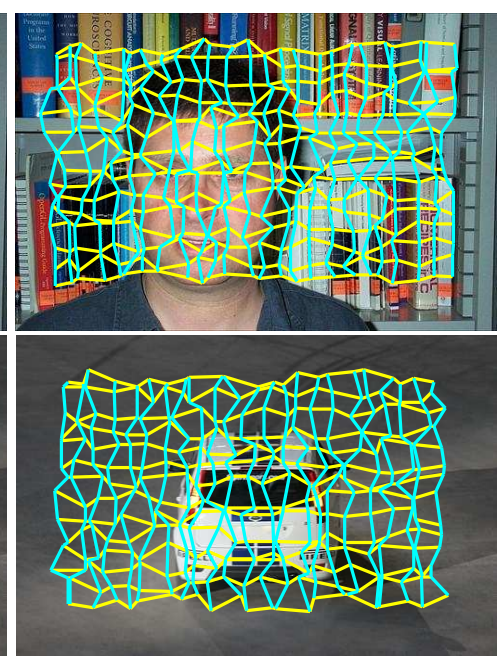

dense interest points

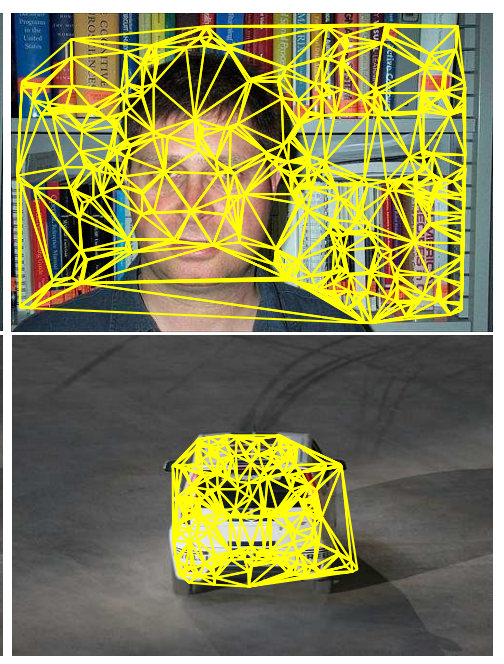

interest points

Figure 5. The grid structure of the regular dense sampling that was used as initialization (left) can be transferred to the dense interest points (middle) and provides spatial structure information such as neighbours. (vertices correspond to the center of extracted patches; only a single scale layer for clarity). For standard interest points (here Hessian Laplace, right), one can compute a Delaunay triangulation, but this sometimes connects far away points and lacks regularity.

the same level of repeatability as standard interest points. This comes as no surprise, since on more or less homogeneous areas in the image, our dense interest point detector is forced to make a selection, even though there is no information available to ensure a stable and repeatable selection. This is especially noticeable for the Bark sequence, where contrast is often relatively low. However, they score significantly better than the features found by dense sampling. When repeatability is the sole criterion (e.g. when the goal is to find correspondences between images), there is no doubt that standard interest points are to be preferred over our dense interest points. However, when representative features are sought for, dense interest points may be a good choice, as they select repeatable features where possible, combined with unstable, yet still informative features elsewhere.

\subsection{Classification}

Next, we evaluate the performance of our dense interest points in the context of a classification task. Here, we focus on a comparison with dense sampling on a regular grid, as this strategy has been shown to outperform interest points in this context (see e.g. [8] or the feature comparison performed in the context of the CVPR09 Features workshop [1] ). As in [1], we use the Pascal VOC2007 dataset [5]. All features are first vector quantized. For this, we use $k$-means run on a subset of the data. Already at this point, we can observe a difference between dense interest points and dense sampling on a regular grid: for the same number of features and the same number of clusters, the obtained clusters are

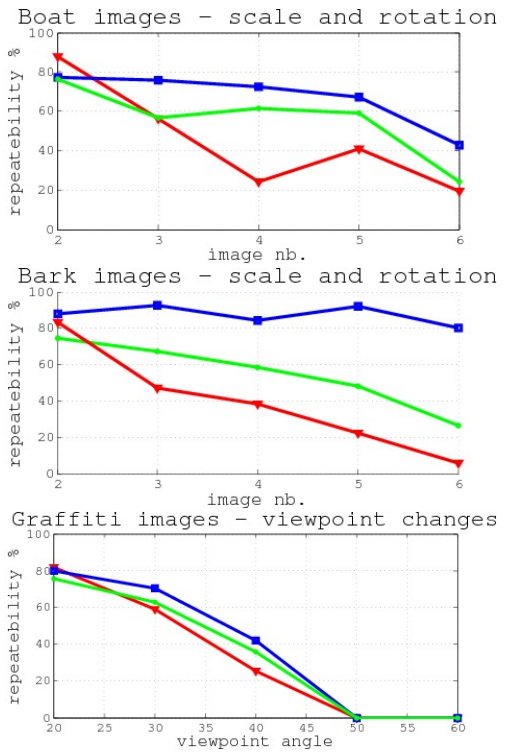

Figure 6. Repeatability of dense interest points (green diamonds), dense sampling on a regular grid (red triangles), and Hessian Laplace (blue squares), for the Boat (scale+rotation), Bark (scale+rotation) and Graffiti (viewpoint changes) sequences.

more compact for the dense interest points, with the sum of squared error output of the clustering algorithm about 5 $10 \%$ smaller.

We experimented with visual vocabularies of size 500, 1000, 2000, and 4000. In most cases, the best results were obtained with the largest vocabularies. Images are repre- 


\begin{tabular}{|l|c|c|c|c|c|c|c|}
\hline & aeroplane & bicycle & bird & boat & bottle & bus & car \\
\hline dense sampling & 61.99 & 47.44 & 30.62 & 46.19 & 22.68 & 37.93 & 61.56 \\
dense interest points & $\mathbf{6 3 . 7 5}$ & $\mathbf{4 9 . 2 4}$ & $\mathbf{3 1 . 8 3}$ & $\mathbf{5 0 . 0 2}$ & $\mathbf{2 3 . 2 3}$ & 37.64 & $\mathbf{6 3 . 1 9}$ \\
\hline \hline & cat & chair & cow & diningtable & dog & horse & motorbike \\
\hline dense sampling & 37.01 & 43.90 & 23.32 & 28.18 & $\mathbf{3 3 . 3 0}$ & 57.00 & 47.04 \\
dense interest points & $\mathbf{3 9 . 6 8}$ & $\mathbf{4 4 . 7 6}$ & $\mathbf{2 9 . 0 8}$ & $\mathbf{3 1 . 3 5}$ & 31.48 & $\mathbf{5 9 . 9 3}$ & 47.22 \\
\hline \hline & person & pottedplant & sheep & sofa & train & tvmonitor & avg \\
\hline dense sampling & 74.70 & 13.87 & 19.94 & 35.47 & 52.39 & 31.92 & 40.32 \\
dense interest points & $\mathbf{7 5 . 3 5}$ & 13.78 & $\mathbf{3 0 . 7 4}$ & 35.53 & $\mathbf{5 7 . 5 2}$ & $\mathbf{3 7 . 1 9}$ & $\mathbf{4 2 . 6 3}$ \\
\hline
\end{tabular}

Table 1. Results on the Pascal VOC 2007 data (measured by average precision).

sented with a bag-of-features representation, ignoring any spatial information. Then, a support vector machine is trained (using [3]), with a $\chi^{2}$-kernel and determining the optimal parameters on the validation set. The results, measured by average precision following the Pascal VOC2007 protocol, are summarized in table 1.

For 15 out of the 20 classes, dense interest points outperform the dense sampling. Only for dogs does dense sampling perform better than dense interest points. Averaged out over all 20 classes, we get an increase in mean average precision of more than $2 \%$ compared to dense sampling. We realize these results are inferior to the winning schemes in the Pascal VOC challenge. However, we used a very standard, baseline scheme (not using any spatial information, not using multiple kernels, etc.). The purpose of this experiment is to compare the performance of the different image representations on a standard dataset, not to compete with these more complex systems. In fact, our results are quite competitive when compared to those reported in the study performed at the CVPR 2009 features workshop [1]. We would probably rank among the top five best performing methods, and are probably the only scheme scoring so high without using color information or extracting significantly larger numbers of features.

\subsection{Matching}

Finally, we show some preliminary results on an image matching task. Recently, Liu et al. have proposed SIFTflow, a scheme to align different images of similar scenes [11]. For each pixel in the image, they compute a SIFT-descriptor. Then, an optical flow like algorithm is used to bring the images into alignment. This works amazingly well, as long as the two images are sufficiently similar ('dense sampling in the space of world images', as they call it). Still, their approach has some limitations. To make it work, SIFT descriptors need to be extracted at each and every pixel in the image. At the same time, to keep the memory requirements under control, the total number of descriptors cannot be too large. To this end, they downscale the image significantly. This results in a loss of detail (smoothing). Moreover, the extracted descriptors end up covering a relatively large part of the image, often containing parts of different scene objects. Finally, descriptors are only computed at a single scale. Changes in scale can only be dealt with in as far as they can be covered by the robustness that comes with SIFT. These constraints are fine when the two images indeed have very similar scene compositions. However, they may be broken when the difference between the images gets larger. The obtained results are good enough for computing a precise scene similarity score [11] or for transferring annotations [10]. However, the warped images look rather patchy, with artefacts due to discontinuities in the flow field.

Instead, we propose to extract dense interest points on the original image (not downscaled). The precise position and scale of each feature (within a certain range) are determined based on the image content. Descriptors can make use of all the information present in the image, as there is no need for smoothing or downscaling. Also, they can be kept relatively small (the same size as typically used in object recognition experiments), limiting the disturbing effect of occlusions or background objects. Some preliminary results are shown in figure 7 . These results were obtained using the code provided by Liu et al., replacing their SIFT computation by our dense interest point computation. We did not use the hierarchical extension, as in our experiments this had a negative effect on the quality of the result. Clearly, the use of dense interest points allows finer detail of the image to be preserved and obtain a better reconstruction.

\section{Conclusion and Future Work}

In this paper we have introduced dense interest points, a hybrid scheme in between dense sampling on a regular grid and interest point detection. We start from image patches sampled on a regular grid, but then refine their position and scale by optimizing an 'interestingness' measure (in our implementation the Laplacian). This results in a set of interest points on a semi-regular grid, densely covering the entire image as is the case with dense sampling, but with repeatability properties closer to those of standard interest points.

Several extensions and variations on this basic scheme 


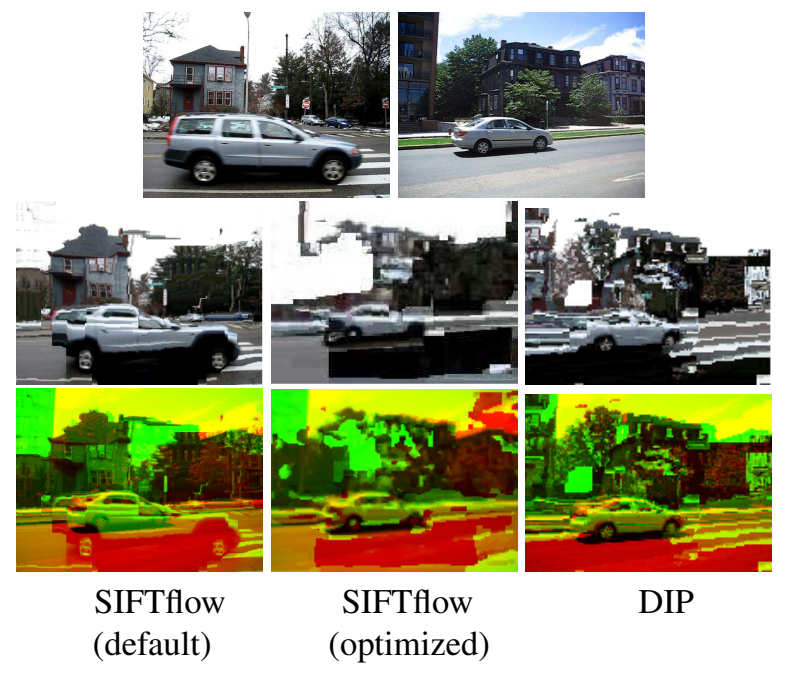

Figure 7. Image matching results. 1st row: source and target image. 2nd and 3rd row: warped images and overlapping images using SIFTflow [11] with default parameters, with parameters optimized for this image pair, and using dense interest points. For the overlap images, the red channel corresponds to the target image, while the green channel corresponds to the warped image.

are possible and worth further investigation. This includes experimenting with more isotropic grid structures as well as other measures for 'interestingness'. One could even integrate different measures, selecting the most appropriate one on a per-patch basis. Also adding a weighting term so as to penalize large grid deformations might be interesting for some applications. Finally, apart from optimizing the location and scale, affine refinements could also be tried.

In future work, we plan to explore how this new image representation can be exploited fully, building on the grid structure that links the features and roughly models the spatial configuration. Also the use of composite features, that are formed by a dense interest point and its immediate neighbours, is worth further investigation. These might be the answer to a longstanding limitation of interest points: on the one hand, they have to be as large as possible, since this increases the distinctiveness. On the other hand, with larger features the assumption that the underlying surface is planar is often violated. Complex features composed of neighbouring dense interest points might provide the right tradeoff between distinctiveness and robustness to non-planarity, since they adapt, to some extent, to local deformations.

Acknowledgements This work was partly funded via the ERC starting independent researcher grant COGNIMUND.

\section{References}

[1] M. Barnard and K. Mikolajczyk. Feature detectors and descriptors: The state of the art and beyond, evaluation. http://www.featurespace.org/.

[2] M. Brown, R. Szeliski, and S. Winder. Multi-image matching using multi-scale oriented patches. In CVPR, pages 510517, 2005.

[3] C.-C. Chang and C.-J. Lin. LIBSVM: a library for support vector machines, 2001. http://www.csie.ntu.edu.tw/ cjlin/libsvm.

[4] B. T. E. Nowak, F. Jurie. Sampling strategies for bag-offeatures image classification. In ECCV, 2006.

[5] M. Everingham, L. Van Gool, C. K. I. Williams, J. Winn, and A. Zisserman. The PASCAL Visual Object Classes Challenge 2007 (VOC2007) Results.

[6] L. Fei-Fei and P. Perona. A bayesian hierarchical model for learning natural scene categories. In CVPR, 2005.

[7] R. Fergus, P. Perona, and A. Zisserman. Weakly supervised scale-invariant learning of models for visual recognition. IJCV , 71(3):273-303, 2007.

[8] C. S. K.E.A. van de Sande, T. Gevers. A comparison of color features for visual concept classification. In ACM Conference on Image and Video Retrieval, 2008.

[9] B. Leibe, A. Leonardis, and B.Schiele. Robust object detection with interleaved categorization and segmentation. IJCV, 77(1-3):259-289, 2008.

[10] C. Liu, J. Yuen, and A. Torralba. Nonparametric scene parsing: label transfer via dense scene alignment. In $C V P R$, 2009.

[11] C. Liu, J. Yuen, A. Torralba, J. Sivic, and W. T. Freeman. Sift flow: dense correspondence across different scenes. In ECCV, 2008. http://people.csail.mit.edu/celiu/ECCV2008/.

[12] D. Lowe. Distinctive image features from scale-invariant keypoint. IJCV, 2(60):91-110, 2004.

[13] R. Marée, P. Geurts, J. Piater, and L. Wehenkel. Random subwindows for robust image classification. In CVPR, 2005.

[14] K. Mikolajczyk and C. Schmid. Scale and affine invariant interest point detectors. IJCV, 1(60):63-86, 2004.

[15] K. Mikolajczyk, T. Tuytelaars, C. Schmid, A. Zisserman, J. Matas, F. Schaffalitzky, T. Kadir, and L. Van Gool. A comparison of affine region detectors. IJCV, 65(1-2):43-72, 2005.

[16] M. Pollefeys. Automatic 3D Modeling with a Hand-Held Camera Images, 2004. ISPRS 2004/3DPVT 2004 tutorial.

[17] X. Ren and J. Malik. Learning a classification model for segmentation. IJCV , 1:10-17, 2003.

[18] J. Sivic, F. Schaffalitzky, and A. Zisserman. Object level grouping for video shots. IJCV, 67(2):189-210, 2006.

[19] J. Sivic and A. Zisserman. Video Google: A text retrieval approach to object matching in videos. In ICCV, 2003.

[20] E. Tola, V. Lepetit, and P. Fua. A fast local descriptor for dense matching. In $C V P R, 2008$.

[21] T.Quack, V. Ferrari, B. Leibe, and L. V. Gool. Efficient mining of frequent and distinctive feature configurations. In ICCV, 2007.

[22] T. Tuytelaars and K. Mikolajczyk. Local invariant feature detectors: A survey. Foundations and Trends in Computer Graphics and Vision, 3(3):177-280, 2008.

[23] T. Tuytelaars and C. Schmid. Vector quantizing feature space with a regular lattice. In $I C C V, 2007$. 